\title{
METRIC THICKENINGS OF EUCLIDEAN SUBMANIFOLDS
}

\author{
HENRY ADAMS AND JOSHUA MIRTH
}

\begin{abstract}
Given a sample $Y$ from an unknown manifold $X$ embedded in Euclidean space, it is possible to recover the homology groups of $X$ by building a Vietoris-Rips or Čech simplicial complex on top of the vertex set $Y$. However, these simplicial complexes need not inherit the metric structure of the manifold, in particular when $Y$ is infinite. Indeed, a simplicial complex is not even metrizable if it is not locally finite. We instead consider metric thickenings, called the Vietoris-Rips and $\breve{C}$ ech thickenings, which are equipped with the 1-Wasserstein metric in place of the simplicial complex topology. We show that for Euclidean subsets $X$ with positive reach, the thickenings satisfy metric analogues of Hausmann's theorem and the nerve lemma (the metric Vietoris-Rips and Čech thickenings of $X$ are homotopy equivalent to $X$ for scale parameters less than the reach). To our knowledge this is the first version of Hausmann's theorem for Euclidean submanifolds (as opposed to Riemannian manifolds), and our result also extends to non-manifold shapes (as not all sets of positive reach are manifolds). In contrast to Hausmann's original proof, our homotopy equivalence is a deformation retraction, is realized by canonical maps in both directions, and furthermore can be proven to be a homotopy equivalence via simple linear homotopies from the map compositions to the corresponding identity maps.
\end{abstract}

\section{INTRODUCTION}

The Vietoris-Rips simplicial complex $\operatorname{VR}(X ; r)$ of a metric space $X$ at scale parameter $r>0$ has $X$ as its vertex set, and a simplex $\sigma$ for every finite set of points of diameter less than $r$. Vietoris-Rips complexes are a natural way to enlarge a metric space. Indeed, Hausmann proves in [18, that given a compact Riemannian manifold $X$ and a sufficiently small scale parameter $r$, the Vietoris-Rips complex $\operatorname{VR}(X ; r)$ is homotopy equivalent to $X$. In response to a question in Hausmann's paper, Latschev [21] proves furthermore that if $Y \subseteq X$ is a sufficiently dense sample, then $\operatorname{VR}(Y ; r)$ is also homotopy equivalent to $X$. Latschev's result is a precursor to many theoretical guarantees [5, 7, 9, 10, 23] showing how Vietoris-Rips complexes and related constructions can recover topological information about a shape $X$ from a sufficiently dense sampling $Y$. In applications of topology to data analysis [8, 13, the datasets will typically be finite, but nevertheless infinite Vietoris-Rips constructions are important for applications in part because if a dataset $Y$ converges to an infinite shape $X$ in the Gromov-Hausdorff distance, then the persistent homology of $\operatorname{VR}(Y ; r)$ converges to that of the infinite object $\operatorname{VR}(X ; r) 9$.

Despite theoretical guarantees such as Hausmann's theorem, the simplicial complex $\operatorname{VR}(X ; r)$ does not retain the metric properties of $X$. In fact, a simplicial complex is metrizable if and only if it is locally finite, which $\operatorname{VR}(X ; r)$ need not be when $X$ is infinite. Furthermore, if $X$ is not discrete then the natural inclusion map $X \hookrightarrow \operatorname{VR}(X ; r)$ is not continuous for any $r>0$. The Vietoris-Rips thickening of $X$, denoted $\operatorname{VR}^{m}(X ; r)$ and introduced in [3], addresses each of these issues. As a set, $\mathrm{VR}^{m}(X ; r)$ is naturally identified with the geometric realization of simplicial complex $\operatorname{VR}(X ; r)$, but it has a completely different topology induced from the 1-Wasserstein metric. Indeed, the space $\operatorname{VR}^{m}(X ; r)$ is a metric thickening of $X$, meaning that it is

Date: April 4, 2018.

2010 Mathematics Subject Classification. 53C23, 54E35, 55P10, 55U10.

Key words and phrases. Vietoris-Rips complex, Čech complex, metric thickening, metric reconstruction, Euclidean submanifold, positive reach, homotopy, clique complex, nerve lemma, persistent homology. 
a metric space extending the metric on $X$. As a result, the inclusion $X \hookrightarrow \mathrm{VR}^{m}(X ; r)$ is continuous for all metric spaces $X$ and scale parameters $r$. In general, the simplicial complex $\operatorname{VR}(X ; r)$ and metric thickening $\mathrm{VR}^{m}(X ; r)$ are neither homeomorphic nor homotopy equivalent, and we argue that the metric thickening is often a more natural object.

In particular, let $X$ be a compact Riemannian manifold. If $X$ is of dimension at least one, then the inclusion $X \hookrightarrow \operatorname{VR}(X ; r)$ is not continuous. For $r$ sufficiently small, the homotopy equivalence $\operatorname{VR}(X ; r) \stackrel{\simeq}{\rightarrow} X$ in Hausmann's result depends on the choice of a total ordering ${ }^{1}$ of the points in $X$, meaning it is non-canonical as different choices of orderings produce different maps. By contrast, the inclusion $X \hookrightarrow \mathrm{VR}^{m}(X ; r)$ to the metric thickening is continuous, and for $r$ sufficiently small it has as a homotopy inverse the canonical map $\operatorname{VR}^{m}(X ; r) \rightarrow X$ defined by Karcher means [3, 19].

In this work we prove a metric analogue of Hausmann's result for subsets of Euclidean space with positive reach. Our main result is the following:

Theorem 3.4. Let $X$ be a subset of Euclidean space $\mathbb{R}^{n}$, equipped with the Euclidean metric, and suppose the reach $\tau$ of $X$ is positive. Then for all $r<\tau$, the metric Vietoris-Rips complex $\operatorname{VR}^{m}(X ; r)$ is homotopy equivalent to $X$.

In particular, if $X$ is a submanifold of $\mathbb{R}^{n}$ with positive reach, then its Vietoris-Rips thickening is homotopy equivalent to $X$ for sufficiently small scale parameters. To our knowledge, this is the first version of Hausmann's theorem for Euclidean (and hence typically non-Riemannian) manifolds, using either VietorisRips complexes or Vietoris-Rips thickenings. However, our theorem does not require $X$ to be a manifold, and indeed not every set of positive reach is a manifold [15, 24].

We prove the main theorem by showing that the linear projection of $\mathrm{VR}^{m}(X ; r)$ into $\mathbb{R}^{n}$ has image contained in the tubular neighborhood of radius $\tau$ about $X$. We then map each point in the tubular neighborhood to its unique closest point in $X$. The composition of these maps produces a homotopy equivalence $\mathrm{VR}^{m}(X ; r) \stackrel{\simeq}{\rightarrow} X$ whose homotopy inverse is the (now continuous) inclusion $X \hookrightarrow \mathrm{VR}^{m}(X ; r)$.

We provide the following motivation for our work. Given a sample $Y$ from an unknown shape $X$, there are theoretical guarantees [5, 7, 9, 10, 21, 23] which recover topological information about $X$ by building a Vietoris-Rips complex or a related construction on top of $Y$. However, many of these theorems require choosing a scale parameter (such as $r$ in $\operatorname{VR}(Y ; r)$ ) which is sufficiently small depending on the curvature of $X$, and hence it may be difficult to choose an appropriate $r$ without prior knowledge of $X$. Instead, practitioners often compute the persistent homology of $\operatorname{VR}(Y ; r)$ over a range of scale parameters $r$ in order to learn about $X$. This is well-motivated since as a dataset $Y$ converges to an infinite shape $X$ in the Gromov-Hausdorff distance, stability implies that the persistent homology of $\operatorname{VR}(Y ; r)$ converges to that of $\operatorname{VR}(X ; r)$ 9. Nevertheless, very little is known about the theory of Vietoris-Rips complexes of infinite shapes (such as manifolds) at larger scale parameters $r$. This is in part due to the difficulty of working with the simplicial complex $\operatorname{VR}(X ; r)$, which may not be metrizable even though $X$ is a metric space, and for which the natural inclusion map $X \hookrightarrow \operatorname{VR}(X ; r)$ is often not continuous. We believe that the metric Vietoris-Rips thickening $\mathrm{VR}^{m}(X ; r)$ is often a more natural object to consider when $X$ is infinite. As evidence of this claim, we provide a metric analogue of Hausmann's theorem $\left(\mathrm{VR}^{m}(X ; r) \simeq X\right.$ for $r$ sufficiently small) when $X$ is a Euclidean subset of positive reach. Our results are relevant for data analysis since a finite dataset $Y$ may converge (as more samples are drawn) in the Gromov-Hausdorff distance to an underlying Euclidean set $X$, and since one may want to recover not only the homotopy type or persistent homology of $X$ but also its metric properties.

\footnotetext{
${ }^{1}$ One could use the axiom of choice to pick such a total order, though constructive total orders may also exist.
} 
An important clarifying remark about metrics on Euclidean submanifolds is the following. Let $X$ be a manifold embedded in Euclidean space $\mathbb{R}^{n}$ and equipped with the Euclidean submetric (as in Theorem 3.4). A different metric on $X$ is the Riemannian structure inherited from the usual inner product in $\mathbb{R}^{n}$-assuming $X$ is connected, the Riemannian distance on $X$ is also a metrization of the original manifold topology [22]. The Vietoris-Rips complex, and its homotopy type, may depend upon which of these two metrics one chooses to use. For example, a circle and an ellipse in $\mathbb{R}^{2}$ with the Riemannian distance function (i.e. the arc-length metric) and equal circumferences have identical Vietoris-Rips complexes. On the other hand, with the Euclidean metrics their Vietoris-Rips complexes are not homotopy equivalent at all scale parameters 2, 4]. The Vietoris-Rips thickening $\operatorname{VR}^{m}(X ; r)$ will similarly depend on the choice of metric on $X$; in this paper we consider the Euclidean metric.

A related construction to the Vietoris-Rips complex is the Čech complex. For $X \subseteq \mathbb{R}^{n}$, the Čech complex $\check{\mathrm{C}}(X ; r)$ is the nerve simplicial complex of the collection of balls $\mathrm{B}(x, r / 2)$ with centers $x \in X$. The nerve lemma implies that $\check{\mathrm{C}}(X ; r)$ is homotopy equivalent to the union of the balls ${ }^{2}$ However, the Cech complex $\check{\mathrm{C}}(X ; r)$ need not inherit any metric properties of $X$, and again is not metrizable if it is not locally finite. We therefore consider the metric Cech thickening $\check{C}^{\mathrm{m}}(X ; r)$ from [3], which is a metric space equipped with the 1-Wasserstein distance that furthermore is a metric thickening of $X$. In Theorem 4.5 we prove that if $X$ is a subset of Euclidean space of positive reach $\tau$, then for all $r<\tau$ the metric Čech thickening $\check{\mathrm{C}}(X ; 2 r)$ is homotopy equivalent to $X{ }^{3}$ The proof mirrors that of the main theorem, Theorem 3.4 .

In Section 2 we review notation and we introduce the Wasserstein metric, Vietoris-Rips and Cech simplicial complexes, Vietoris-Rips and Čech metric thickenings, Euclidean subsets of positive reach, and tubular neighborhoods. Section 3 contains our main result, a metric analogue of Hausmann's theorem for VietorisRips thickenings of Euclidean subsets of positive reach, and the lemmas building up to it. We use similar techniques to prove a version for Čech thickenings in Section 4

\section{Preliminaries}

We describe background material and notation on metric spaces, Euclidean space, topological spaces 17, the Wasserstein metric [28, 14], simplicial complexes, Vietoris-Rips and Čech simplicial complexes [13, Vietoris-Rips and Čech metric thickenings [3], and sets of positive reach [15].

2.1. Metric spaces. A metric space, $(X, d)$, is a set $X$ along with a function $d: X \times X \rightarrow \mathbb{R}$ such that for all $u, v, w \in X$,

- $d(u, v) \geq 0$ and $d(u, v)=0$ if and only if $u=v$,

- $d(u, v)=d(v, u)$, and

- $d(u, v) \leq d(u, w)+d(w, v)$.

The function $d$ is called a distance or a metric. We will denote open balls in $X$ by $\mathrm{B}(x, r)=\{y \in X \mid d(y, x)<$ $r$, where $x \in X$ is the ball's center and $r$ is its radius. Likewise, a closed ball will be denoted $\overline{\mathrm{B}}(x, r)=$ $\{y \in X \mid d(y, x) \leq r\}$.

Given a point $x \in X$ and subset $Y \subseteq X$, we define the distance between $x$ and $Y$ to be $d(x, Y)=$ $\inf _{y \in Y} d(x, y)$. The distance between two subsets $Y, Y^{\prime} \subseteq X$ is $d\left(Y, Y^{\prime}\right)=\inf _{y \in Y, y^{\prime} \in Y^{\prime}} d\left(y, y^{\prime}\right)$. An $r$ thickening of a metric space $X$ is a metric space $Z \supseteq X$ such that the metric on $X$ extends to that on $Z$, and

\footnotetext{
${ }^{2}$ Here we mean ambient Čech complexes corresponding to Euclidean balls, though in this paper we also consider intrinsic Čech complexes corresponding to possibly non-contractible balls in $X$.

${ }^{3}$ This result does not follow from the nerve lemma since the nerve complex $\breve{\mathrm{C}}(X ; r)$ and metric thickening $\check{\mathrm{C}}^{\mathrm{m}}(X ; r)$ can in general have different homotopy types. The theorem holds for both ambient and intrinsic Čech thickenings.
} 
also $d(x, Z) \leq r$ for all $x \in X$. We define the diameter of a set $Y \subseteq X$ to be $\operatorname{diam}(Y)=\sup \left\{d\left(y, y^{\prime}\right) \mid y, y^{\prime} \in\right.$ $Y\}$.

Any metric $d$ induces a topology on the set $X$ called the metric topology. The basis for this topology consists of all open balls of positive radius. A topological space $X$ is metrizable if there exists a metric $d: X \times X \rightarrow \mathbb{R}$ that induces the topology of $X$.

2.2. Euclidean Space. Euclidean space is the metric space $\left(\mathbb{R}^{n}, d\right)$ where $d$ is the usual Euclidean distance. There is a standard inner product $\langle\cdot, \cdot\rangle$ on $\mathbb{R}^{n}$ defined by

$$
\left\langle\left(u_{1}, \ldots, u_{n}\right),\left(v_{1}, \ldots, v_{n}\right)\right\rangle=u_{1} v_{1}+\ldots+u_{n} v_{n} .
$$

We can define the norm, $\|\cdot\|$, of an element $x \in \mathbb{R}^{n}$ by $\|x\|=\langle x, x\rangle^{1 / 2}$. The metric $d$ is then simply $d(u, v)=\|u-v\|$.

For any $X \subseteq \mathbb{R}^{n}$ define the convex hull of $X$ as

$$
\operatorname{conv}(X)=\left\{x=\sum_{i=0}^{k} \lambda_{i} x_{i} \in \mathbb{R}^{n} \mid k \in \mathbb{N}, \lambda_{i} \geq 0, \sum_{i} \lambda_{i}=1, x_{i} \in X\right\} .
$$

Note that the diameter of $X$ is the same as the diameter of its convex hull. If $X \subseteq \mathbb{R}^{n}$, we denote by $X^{c}$ the complement of $X$, that is, $X^{c}=\mathbb{R}^{n} \backslash X$, and by $\bar{X}$ the closure of $X$ in $\mathbb{R}^{n}$.

2.3. Topological spaces. Let $Y$ and $Z$ be topological spaces. Two continuous maps $f, g: Y \rightarrow Z$ are homotopy equivalent if there exists a continuous map $H: Y \times I \rightarrow Z$ with $H(\cdot, 0)=f$ and $H(\cdot, 1)=g$, where $I=[0,1]$ is the unit interval [17. Two spaces $Y$ and $Z$ are homotopy equivalent if there exist continuous maps $f: Y \rightarrow Z$ and $g: Z \rightarrow Y$ such that $g \circ f$ is homotopy equivalent to the identity $\operatorname{map} \operatorname{id}_{Y}$ on $Y$, and

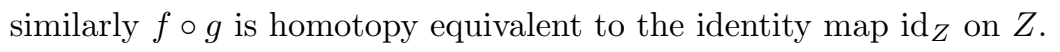

2.4. Wasserstein metric. In this section we describe a way to put a metric on probability Radon measures. The metric has many names: the Wasserstein, Kantorovich, optimal transport, or earth mover's metric. It is known to solve the Monge-Kantorovich problem (see [28]).

Let $X$ be a metric space equipped with a distance function $d: X \times X \rightarrow \mathbb{R}$. A measure $\mu$ defined on the Borel sets of $X$ is

- inner regular if $\mu(B)=\sup \{\mu(K) \mid K \subseteq B$ is compact $\}$ for all Borel sets $B$,

- locally finite if every point $x \in X$ has a neighborhood $U$ such that $\mu(U)<\infty$,

- a Radon measure if it is both inner regular and locally finite, and

- a probability measure if $\int_{X} d \mu=1$.

The following is from [14, 20]. Let $\mathcal{P}(X)$ denote the set of probability Radon measures such that for some (and hence all) $y \in X$, we have $\int_{X} d(x, y) d \mu<\infty$. Define the $L^{1}$ metric on $X \times X$ by setting the distance between $\left(x_{1}, x_{2}\right),\left(x_{1}^{\prime}, x_{2}^{\prime}\right) \in X \times X$ to be $d\left(x_{1}, x_{1}^{\prime}\right)+d\left(x_{2}, x_{2}^{\prime}\right)$. Given $\mu, \nu \in \mathcal{P}(X)$, let $\Pi(\mu, \nu) \subseteq \mathcal{P}(X \times X)$ be the set of all probability Radon measures $\pi$ on $X \times X$ such that $\mu(B)=\pi(B \times X)$ and $\nu(B)=\pi(X \times B)$ for all Borel subsets $B \subseteq X$. Note that such an element $\pi$ is a joint measure on $X \times X$ whose marginals, when restricted to each $X$ factor, are $\mu$ and $\nu$.

Definition 2.1. The 1-Wasserstein metric on $\mathcal{P}(X)$ is defined by

$$
d_{W}(\mu, \nu)=\inf _{\pi \in \Pi(\mu, \nu)} \int_{X \times X} d(x, y) d \pi .
$$

The names optimal transport or earth mover's metric can be interpreted as follows. One can think of measures $\mu$ and $\nu$ as "piles of dirt" in $X$ with prescribed mass distributions. The joint measure $\pi$ with $\mu$ and $\nu$ as marginals is a transport plan moving the $\mu$ pile of dirt to the $\nu$ pile. The 1 -Wasserstein distance between 
$\mu$ and $\nu$ is the infimum, over all transport plans $\pi$, of the work involved in moving $\mu$ to $\nu$ via transport plan $\pi$.

\subsection{Simplicial Complexes.}

Definition 2.2. Let $V$ be a set, called the set of vertices. An abstract simplicial complex $K$ on vertex set $V$ is a subset of the power set of $V$ with the property that if $\sigma \in K$, then all subsets of $\sigma$ are in $K$.

Every abstract simplicial complex permits a geometric realization, $|K|$, which is a topological space. As a set we have

$$
|K|=\left\{\sum_{i=0}^{k} \lambda_{i} v_{i} \mid k \in \mathbb{N}, \lambda_{i} \geq 0, \sum_{i} \lambda_{i}=1,\left[v_{0}, \ldots, v_{k}\right] \in K\right\} .
$$

In the simpler case when $K$ is finite, we can put a topology on $|K|$ as follows. Choose an affinely independent set of points in $\mathbb{R}^{n}$ (for $n$ sufficiently large) to correspond to each of the elements of the vertex set $V$. Then $|K|$ consists of all convex linear combinations of these points, and $|K|$ is given its topology as a subset of Euclidean space. More generally, one can produce a topology on $|K|$ by viewing it as a subset of $[0,1]^{V}$, the space of functions $V \rightarrow[0,1]$. Indeed note

$$
|K|=\left\{f:[0,1] \rightarrow V \mid \sum_{v \in V} f(v)=1, \operatorname{supp}(f) \in K\right\} .
$$

Give $[0,1]^{V}$ its induced topology as the direct limit of $[0,1]^{\tau}$ where $\tau$ ranges over all finite subsets of $V$, and equip $|K|$ with the subspace topology [26].

For the rest of this paper we denote both an abstract simplicial complex and its geometric realization by the same symbol $K$.

2.6. The Vietoris-Rips and Čech simplicial complexes. Two natural ways to enlarge a metric space by building a simplicial complex on top of it are the Vietoris-Rips and Čech complex constructions.

Definition 2.3. Let $X$ be a metric space and $r>0$ a scale parameter. The Vietoris-Rips simplicial complex of $X$ with scale parameter $r$, denoted $\mathrm{VR}_{\leq}(X ; r)$, has vertex set $X$ and a simplex for every finite subset $\sigma \subseteq X$ such that $\operatorname{diam}(\sigma) \leq r$. Similarly, $\mathrm{VR}_{<}(X ; r)$ contains every finite subset with diameter $<r$.
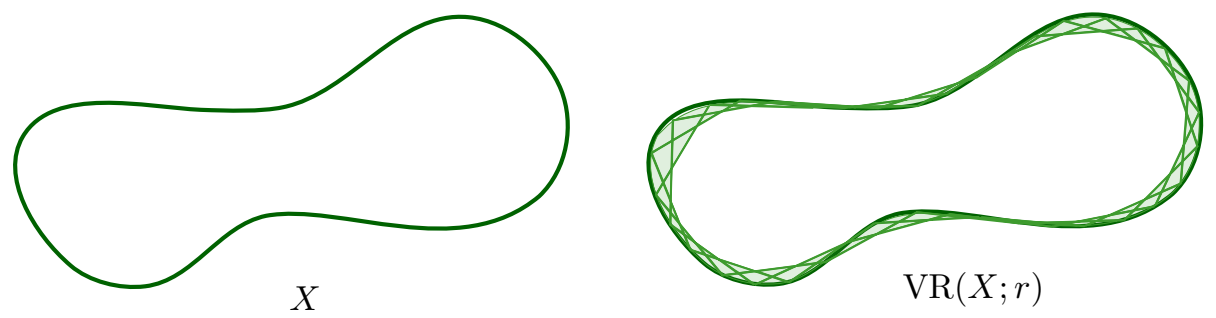

Figure 1. A metric space $X$ and (a subset of) its Vietoris-Rips complex.

We will write $\operatorname{VR}(X ; r)$ when the distinction between $<$ and $\leq$ is unimportant. The Vietoris-Rips complex is the clique or flag complex of its 1-skeleton. 
Definition 2.4. Let $X \subseteq Y$ be a submetric space and $r$ a scale parameter with $r \geq 0$. The Čech complex of $X$ with scale parameter $r, \check{\mathrm{C}}_{\leq}(X, Y ; r)$, has vertex set $X$ and a simplex for every finite subset $\sigma \subseteq X$ such that $\bigcap_{x_{i} \in \sigma} \overline{\mathrm{B}}\left(x_{i}, r / 2\right) \neq \emptyset$, where $\overline{\mathrm{B}}\left(x_{i}, r / 2\right)$ denotes a closed ball in $Y$ centered at $x_{i}$ with radius $r / 2$.

Similarly, $\check{\mathrm{C}}_{<}(X, Y ; r)$ contains a simplex for every finite subset $\sigma$ such that $\bigcap_{x_{i} \in \sigma} \mathrm{B}\left(x_{i}, r / 2\right) \neq \emptyset$.

Again, we will write $\check{C}(X, Y ; r)$ when the distinction between open and closed is unimportant. The Čech complex can be considered as the nerve of the union of balls in $Y$ of radius $r / 2$ centered at each of the points in $X$. Of particular interest are the cases where $Y=\mathbb{R}^{n}$ and $Y=X$. These are called the called the ambient and intrinsic Čech complex, respectively. Note that if $X \subseteq \mathbb{R}^{n}$ then $\check{\mathrm{C}}(X, X ; r) \subseteq \check{\mathrm{C}}\left(X, \mathbb{R}^{n} ; r\right)$. When it is not necessary to distinguish these two we will write $\check{\mathrm{C}}(X ; r)$.

For any $\sigma \in \check{\mathrm{C}}(X, Y ; r)$ we have $\operatorname{diam}(\sigma) \leq r$, and so $\check{\mathrm{C}}(X, Y ; r)$ is a subcomplex of $\operatorname{VR}(X ; r)$. When $Y$ is a geodesic space, the complexes $\operatorname{VR}(X ; r)$ and $\check{\mathrm{C}}(X, Y ; r)$ have the same 1-skeletons. But independent of whether $Y$ is geodesic or not, the Čech complex can be a proper subset of the Vietoris-Rips complex.

A useful characterisation is the following:

Proposition 2.5. A set of points $x_{0}, \ldots, x_{k} \in X$ form a simplex in $\check{\mathrm{C}}_{<}(X, Y ; r)$ if and only if there exists a point $c \in Y$ such that every $x_{i}$ is contained in the open ball $\mathrm{B}(c, r / 2)$. A similar statement is true for $\check{\mathrm{C}}_{\leq}(X, Y ; r)$ with closed balls.

Proof. Let $c$ be any point in the intersection $\bigcap_{x_{i} \in \sigma} B\left(x_{i}, r / 2\right)$. Then $B(c, r / 2)$ contains all of the $x_{i}$. Conversely, if all of the $x_{i}$ are in $B(c, r / 2)$, then $c$ is in $\bigcap_{x_{i} \in \sigma} B\left(x_{i}, r / 2\right)$ and hence the intersection is nonempty.

The Vietoris-Rips and Čech complexes are given the standard topology as simplicial complexes: a subset of the geometric realization is open if and only if its intersection with every simplex is open. An important remark is the following. A simplicial complex $K$ is said to be locally finite if each vertex belongs to only finitely many simplices of $K$, and a simplicial complex is metrizable if and only if it is locally finite [25, Proposition 4.2.16(2)]. This means that in general, the Vietoris-Rips and Čech simplicial complexes cannot be equipped with a metric without changing their homeomorphism types, even though they were built on top of a metric space.

2.7. The Vietoris-Rips and Čech Thickenings. The definitions in this section are from [3, 14, 20,

Given a metric space $X$ and a scale parameter $r$ we will define the Vietoris-Rips thickening $\mathrm{VR}^{m}(X ; r)$, which will be a metric space $r$-thickening of $X$. As a set, $\operatorname{VR}^{m}(X ; r)$ is the set of all formal convex combinations of points in $X$ with diameter at most $r$, namely

$$
\begin{aligned}
& \operatorname{VR}_{\leq}^{m}(X ; r)=\left\{\sum_{i=0}^{k} \lambda_{i} x_{i} \mid k \in \mathbb{N}, \lambda_{i} \geq 0, \sum_{i} \lambda_{i}=1, x_{i} \in X, \text { and } \operatorname{diam}\left(\left\{x_{0}, \ldots, x_{k}\right\}\right) \leq r\right\} \\
& \operatorname{VR}_{<}^{m}(X ; r)=\left\{\sum_{i=0}^{k} \lambda_{i} x_{i} \mid k \in \mathbb{N}, \lambda_{i} \geq 0, \sum_{i} \lambda_{i}=1, x_{i} \in X, \text { and } \operatorname{diam}\left(\left\{x_{0}, \ldots, x_{k}\right\}\right)<r\right\} .
\end{aligned}
$$

A useful viewpoint is to consider an element of $\operatorname{VR}^{m}(X ; r)$ as a probability measure. For $x \in X$, let $\delta_{x}$ be the Dirac probability measure defined on any Borel subset $E \subseteq X$ by

$$
\delta_{x}(E)= \begin{cases}1 & \text { if } x \in E \\ 0 & \text { if } x \notin E .\end{cases}
$$

\footnotetext{
${ }^{4}$ There is a canonical bijection between the sets underlying $\operatorname{VR}^{m}(X ; r)$ and the geometric realization $|\operatorname{VR}(X ; r)|$ of the Vietoris-Rips simplicial complex. However, these two topological spaces will often not be homeomorphic (and sometimes not even homotopy equivalent).
} 
By identifying $x \in X$ with $\delta_{x} \in \mathcal{P}(X)$, and more generally $x=\sum_{i=0}^{k} \lambda_{i} x_{i}$ with $\sum_{i=0}^{k} \lambda_{i} \delta_{x_{i}} \in \mathcal{P}(X)$, we can view $\operatorname{VR}^{m}(X ; r)$ as a subset of $\mathcal{P}(X)$, the set of all Radon probability measures on $X$. Hence we can equip the set $\operatorname{VR}^{m}(X ; r)$ with the 1-Wasserstein metric from Section 2.4 , namely $d_{W}\left(x, x^{\prime}\right)=$ $\inf _{\pi \in \Pi\left(x, x^{\prime}\right)} \int_{X \times X} d\left(x, x^{\prime}\right) d \pi$ for $x, x^{\prime} \in \mathrm{VR}^{m}(X ; r)$.

To give a more explicit definition of the metric on $\mathrm{VR}^{m}(X ; r)$, let $x, x^{\prime} \in \mathrm{VR}^{m}(X ; r)$ with $x=\sum_{i=0}^{k} \lambda_{i} x_{i}$ and $x^{\prime}=\sum_{i=0}^{k^{\prime}} \lambda_{i}^{\prime} x_{i}^{\prime}$ (we cease to distinguish between $x \in X$ and its associated measure, $\delta_{x}$ ). Define a matching $p$ between $x$ and $x^{\prime}$ to be any collection of non-negative real numbers $\left\{p_{i, j}\right\}$ such that $\sum_{j=0}^{k^{\prime}} p_{i, j}=\lambda_{i}$ and $\sum_{i=0}^{k} p_{i, j}=\lambda_{j}^{\prime}$. It follows as a consequence that $\sum_{i, j} p_{i, j}=1$, and so matching $\left\{p_{i, j}\right\}$ can be thought of as a joint probability distribution with marginals $\left\{\lambda_{i}\right\}_{i=0}^{k}$ and $\left\{\lambda_{j}^{\prime}\right\}_{j=0}^{k^{\prime}}$. Define the cost of the matching $p$ to be $\operatorname{cost}(p)=\sum_{i, j} p_{i, j} d\left(x_{i}, x_{j}^{\prime}\right)$.

Definition 2.6. The 1-Wasserstein metric on $\mathrm{VR}^{m}(X ; r)$ is the distance $d_{W}$ defined by

$$
d_{W}\left(x, x^{\prime}\right)=\inf \left\{\operatorname{cost}(p) \mid p \text { is a matching between } x \text { and } x^{\prime}\right\} .
$$

Similar to the Vietoris-Rips thickening, we can construct the Čech thickening $\check{\mathrm{C}}^{\mathrm{m}}(X, Y ; r)$ equipped with the 1-Wasserstein metric. The construction is exactly the same, except that the elements of $\check{\mathrm{C}}^{\mathrm{m}}(X, Y ; r)$ are the convex combinations of vertices from simplices in $\check{\mathrm{C}}(X, Y ; r)$ (rather than in $\operatorname{VR}(X ; r))$. By [3, Lemma 3.5], both $\operatorname{VR}^{m}(X ; r)$ and $\check{C}^{\mathrm{m}}(X, Y ; r)$ are $r$-thickenings of the metric space $X$.

One could alternatively consider a $p$-Wasserstein metric for $1 \leq p \leq \infty$.

2.8. Sets of positive reach. We are interested in the case where metric space $X$ is a subset of $\mathbb{R}^{n}$ of positive reach. In particular, any embedded $C^{k}$ submanifold (with or without boundary) of $\mathbb{R}^{n}$ with $k \geq 2$ has positive reach [27, but not every set of positive reach is a manifold [15, 24]. Consider the set

$$
Y=\left\{y \in \mathbb{R}^{n} \mid \exists x_{1} \neq x_{2} \in X \text { with } d\left(y, x_{1}\right)=d\left(y, x_{2}\right)=d(y, X)\right\} .
$$

The closure $\bar{Y}$ of $Y$ is the medial axis of $X$. For any point $x \in X$, the local feature size at $x$ is the distance $d(x, \bar{Y})$ from $x$ to the medial axis. The reach $\tau$ of $X$ is the minimal distance $\tau=d(X, \bar{Y})$ between $X$ and its medial axis.

For $X \subseteq \mathbb{R}^{n}$ and $\alpha>0$ we define its $\alpha$-offset (or tubular neighborhood), Tub ${ }_{\alpha}$, by

$$
\operatorname{Tub}_{\alpha}=\left\{x \in \mathbb{R}^{n} \mid d(x, X)<\alpha\right\}=\bigcup_{x \in X} \mathrm{~B}(x, \alpha) .
$$

In particular, if $X$ has reach $\tau$, then for every point in $\operatorname{Tub}_{\tau}$ there exists a unique nearest point in $X$. As in [15, 23], define $\pi: \operatorname{Tub}_{\tau} \rightarrow X$ to be the nearest point projection map, sending an element $x \in \mathrm{Tub}_{\tau}$ to its unique closest point $\pi(x) \in X$.

Lemma 2.7. The function $\pi: \operatorname{Tub}_{\tau} \rightarrow X$ is continuous.

Proof. Let $x, y \in \operatorname{Tub}_{\tau}$ and $r=\max \{d(x, \pi(x)), d(y, \pi(y))\}$. Then the conditions of [15, Theorem 4.8(8)] are satisfied and so

$$
d(\pi(x), \pi(y)) \leq \frac{\tau}{\tau-r} d(x, y) .
$$

Thus $\pi$ is continuous at $x$ for any $x \in \operatorname{Tub}_{\tau}$.

We also state the following proposition, implicit in 23, for any set of positive reach.

Proposition 2.8. Let $X \subseteq \mathbb{R}^{n}$ have reach $\tau>0$. Let $p \in X$ and suppose $x \in \operatorname{Tub}_{\tau} \backslash X$ satisfies $\pi(x)=p$.

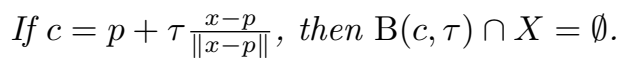


Proof. For any $0<t<\tau$, let $y_{t}=p+t \frac{x-p}{\|x-p\|}$. Since $y_{t} \in \operatorname{Tub}_{\tau}$, we have $\overline{\mathrm{B}}\left(y_{t}, t\right) \cap X=\{p\}$ and $d\left(y_{t}, p\right)=t$, so $\mathrm{B}(c, t) \cap X=\emptyset$. Note that $\mathrm{B}(c, \tau)=\cup_{0<t<\tau} \mathrm{B}\left(y_{t}, t\right)$. Indeed, to see the inclusion $\subseteq$, suppose that $z \in \mathrm{B}(c, \tau)$, so that $d(z, c)=\tau-\epsilon$ for some $\epsilon>0$. Let $t=\tau-\frac{\epsilon}{3}$. By the triangle inequality, $d\left(y_{t}, z\right) \leq d\left(y_{t}, c\right)+d(c, z)=\tau-\frac{2 \epsilon}{3}<t$, giving $z \in \mathrm{B}\left(y_{t}, t\right)$. The reverse inclusion $\supseteq$ is straightforward. It follows that $\mathrm{B}(c, \tau) \cap X=\emptyset$.

\section{A metric and Euclidean analogue of Hausmann's Result}

We now present our main theorem, a metric analogue of Hausmann's result for Vietoris-Rips thickenings of subsets of Euclidean space with positive reach. Since in Section 4 we will also give an analogous theorem for the metric Čech thickening, we provide some notation now for both cases. Let $X \subseteq \mathbb{R}^{n}$ be a set of positive reach. Let $K(X ; r)$ be either a Vietoris-Rips complex or Cech complex of $X$ with scale parameter $r$, and let $K^{m}(X ; r)$ be the corresponding metric Vietoris-Rips or Čech thickening. Define $f: K^{m}(X ; r) \rightarrow \mathbb{R}^{n}$ to be the linear projection map $f\left(\sum_{i} \lambda_{i} x_{i}\right)=\sum_{i} \lambda_{i} x_{i} \in \mathbb{R}^{n}$, where the first sum is a formal convex combination of points in $X$, and the second sum is the standard addition of vectors in $\mathbb{R}^{n}$. Recall $\pi$ : $\operatorname{Tub}_{\tau} \rightarrow X \subseteq \mathbb{R}^{n}$ is the nearest-point projection map.

Several geometric lemmas are required.

Lemma 3.1. Let $x_{0}, \ldots, x_{k} \in \mathbb{R}^{n}$, let $y \in \operatorname{conv}\left\{x_{0}, \ldots, x_{k}\right\}$, and let $C$ be a convex set with $y \notin C$. Then there is at least one $x_{i}$ with $x_{i} \notin C$.

Proof. Suppose for a contradiction that we had $x_{i} \in C$ for all $i=0, \ldots, k$. Then since $C$ is convex, we'd also have $y \in \operatorname{conv}\left\{x_{0}, \ldots, x_{k}\right\} \subseteq C$. Hence it must be the case that $x_{i} \notin C$ for some $i$.

Lemma 3.2. For $X \subseteq \mathbb{R}^{n}$ and $r>0$, the map $f: \operatorname{VR}^{m}(X ; r) \rightarrow \mathbb{R}^{n}$ has its image contained in $\overline{\text { Tub }}$.

Proof. Let $x=\sum_{i=0}^{k} \lambda_{i} x_{i} \in \mathrm{VR}^{m}(X ; r)$; we have

$$
\operatorname{diam}\left(\operatorname{conv}\left\{x_{0}, \ldots, x_{k}\right\}\right)=\operatorname{diam}\left(\left[x_{0}, \ldots, x_{k}\right]\right) \leq r .
$$

Since $f(x) \in \operatorname{conv}\left\{x_{0}, \ldots, x_{k}\right\}$, it follows that $d(f(x), X) \leq d\left(f(x), x_{0}\right) \leq r$, and so $f(x) \in \overline{\operatorname{Tub}_{r}}$.

The substance of Lemma 3.3 will be that if $\left[x_{0}, \ldots, x_{k}\right]$ is a simplex in $\operatorname{VR}(X ; r)$ and if $x=\sum_{i} \lambda_{i} x_{i} \in$ $\mathrm{VR}^{m}(X ; r)$, then $\pi(f(x))$ will be "close enough" to $x_{0}, \ldots, x_{k}$ so that $\left[x_{0}, \ldots, x_{k}, \pi(f(x))\right]$ is also a simplex in $\operatorname{VR}(X ; r)$. This fact will be crucial for defining the homotopy equivalences in our proof of Theorem 3.4 .

Lemma 3.3. Let $X \subseteq \mathbb{R}^{n}$ have positive reach $\tau$, let $\left[x_{0}, \ldots x_{k}\right]$ be a simplex in $\operatorname{VR}(X ; r)$ with $r<\tau$, let $x=\sum \lambda_{i} x_{i} \in \mathrm{VR}^{m}(X ; r)$, and let $p=\pi(f(x))$. Then the simplex $\left[x_{0}, \ldots, x_{k}, p\right]$ is in $\operatorname{VR}(X ; r)$.

Proof. We write the proof for $\mathrm{VR}_{\leq}(X ; r)$; an analogous proof works for $\mathrm{VR}_{<}(X ; r)$. Note $p=\pi(f(x))$ is defined by Lemma 3.2 since $\overline{\operatorname{Tub}_{r}} \subseteq \operatorname{Tub}_{\tau}$. We may assume $p \neq f(x)$, since otherwise the conclusion follows as $f(x)$ is in the convex hull of the $x_{i}$.

Suppose for a contradiction that $d\left(x_{i}, p\right)>r$ for some $i$; without loss of generality we may assume $i=0$. Since $d\left(x_{0}, f(x)\right) \leq r$ we have that $f(x) \neq p$. Following [23], let $c=p+\tau \frac{f(x)-p}{\|f(x)-p\|}$, and let $\mathrm{B}(c, \tau)$ be the open ball of radius $\tau$ that is tangent to $X$ at $p$. By Proposition 2.8 this open ball does not intersect $X$, giving $x_{0}, \ldots, x_{k} \notin \mathrm{B}(c, \tau)$. Define $T_{p}^{\perp}$ to be the line through $f(x)$ and $p$. Since $f(x)$ is between $p$ and $c$ on $T_{p}^{\perp}$, note that $d\left(x_{0}, f(x)\right) \leq r$ implies $x_{0}$ is not on $T_{p}^{\perp}$. Let $x_{0}^{\prime} \neq x_{0}$ be the closest point on $T_{p}^{\perp}$ to $x_{0}$. Let $H_{x_{0}}=\left\{z \in \mathbb{R}^{n} \mid\left\langle z-x_{0}^{\prime}, x_{0}-x_{0}^{\prime}\right\rangle>0\right\}$ be the open half-space containing $x_{0}$, whose boundary is the hyperplane containing $T_{p}^{\perp}$ that's perpendicular to $x_{0}-x_{0}^{\prime}$. Since $d\left(x_{0}, p\right), d\left(x_{0}, c\right)>r$, it follows that $H_{x_{0}}^{c} \cap \overline{\mathrm{B}}\left(x_{0}, r\right) \subseteq \mathrm{B}(c, \tau)$. Since $x_{i} \in \overline{\mathrm{B}}\left(x_{0}, r\right) \backslash \mathrm{B}(c, \tau)$, this implies that $x_{i} \in H_{x_{0}}$ for all $i$. This contradicts 
Lemma 3.1 since $H_{x_{0}}$ is convex with $f(x) \notin H_{x_{0}}$, even though $f(x) \in \operatorname{conv}\left(\left\{x_{0}, \ldots, x_{k}\right\}\right)$. Hence it must be the case that $d\left(x_{0}, p\right) \leq r$, and it follows that $\left[x_{0}, \ldots, x_{k}, p\right]$ is a simplex in $\mathrm{VR}_{\leq}(X ; r)$.

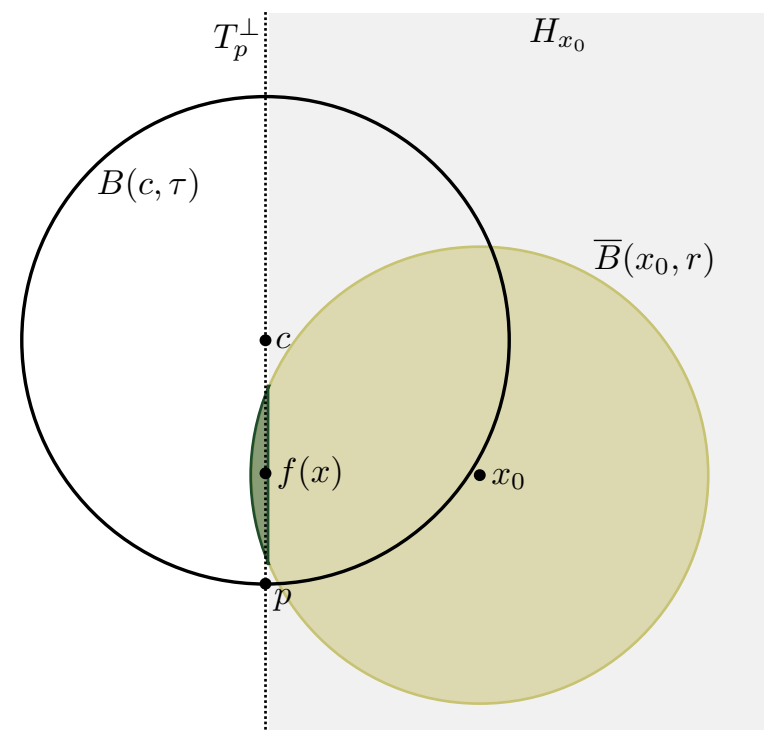

FiguRE 2. Figure for the proof of Lemma 3.3 The green shaded region is a subset of $\mathrm{B}(c, \tau)$, forcing all $x_{i}$ to be in the same half-space.

We are now prepared to prove our main result. We remark that when $X$ is a manifold, it may be possible to estimate the reach of $X$ using the techniques of [1, 6, 12, 16,

Theorem 3.4. Let $X$ be a subset of Euclidean space $\mathbb{R}^{n}$, equipped with the Euclidean metric, and suppose the reach $\tau$ of $X$ is positive. Then for all $r<\tau$, the metric Vietoris-Rips thickening $\operatorname{VR}^{m}(X ; r)$ is homotopy equivalent to $X$.

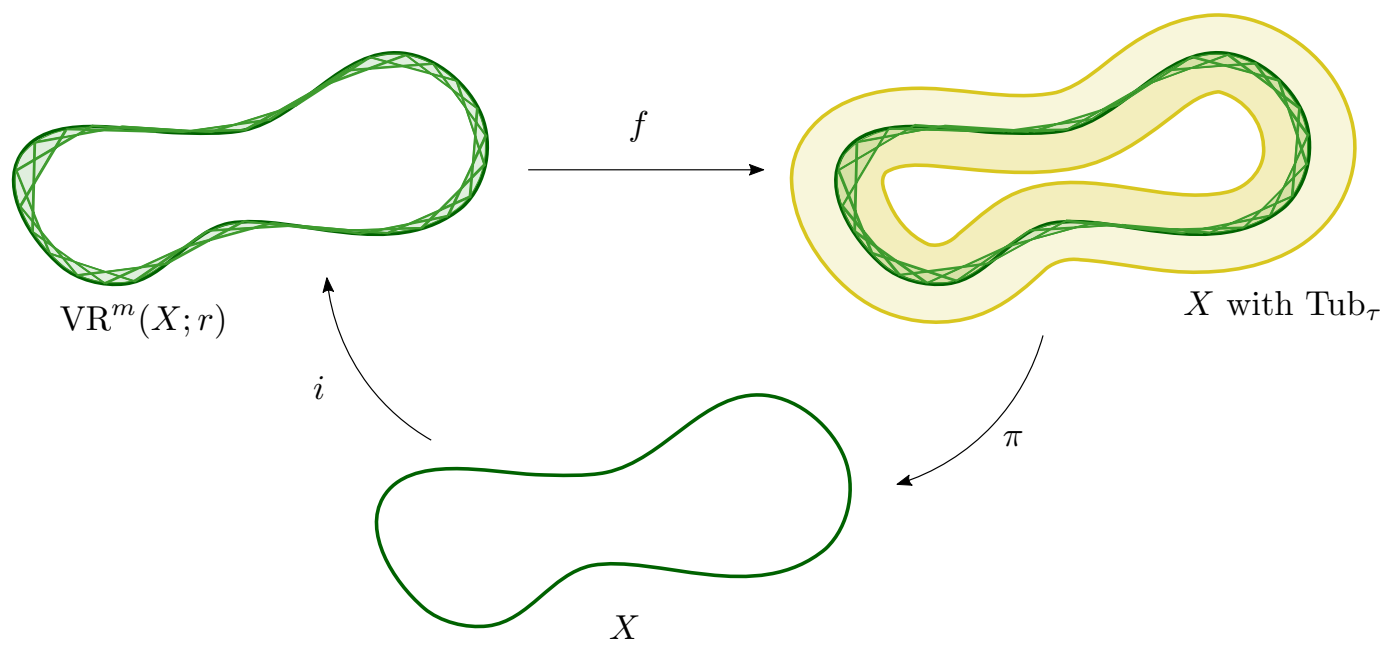

Figure 3. The homotopy equivalence between $\operatorname{VR}^{m}(X ; r)$ and $X$ in Theorem 3.4 
Proof. By [3, Lemma 5.2], map $f: \mathrm{VR}^{m}(X ; r) \rightarrow \mathbb{R}^{n}$ is 1-Lipschitz and hence continuous. It follows from Lemma 3.2 that the image of $f$ is a subset of $\operatorname{Tub}_{\tau}$. By Lemma 2.7 we have that $\pi$ : $\operatorname{Tub}_{\tau} \rightarrow X$ is continuous. Let $\iota: X \rightarrow \mathrm{VR}^{m}(X ; r)$ be the natural inclusion map.

We will show that $\iota$ and $\pi \circ f$ are homotopy inverses. Note that $\pi \circ f \circ i=\operatorname{id}_{X}$. Consider the map $H: \operatorname{VR}^{m}(X ; r) \times I \rightarrow \operatorname{VR}^{m}(X ; r)$ defined by $H(x, t)=t \cdot \operatorname{id}_{\mathrm{VR}^{m}(X ; r)}+(1-t) \iota \circ \pi \circ f$. Map $H$ is well-defined by Lemma 3.3 , and continuous by [3, Lemma 3.8] since $\pi \circ f$ is continuous. It follows that $H$ is a homotopy equivalence from $\iota \circ \pi \circ f$ to $i d_{\mathrm{VR}^{m}(X ; r)}$, and hence $\operatorname{VR}^{m}(X ; r)$ is homotopy equivalent to $X$.

\section{A metric and Euclidean analogue of the nerve lemma}

We handle the case of Čech thickenings in a similar fashion in this section. We write $\check{\mathrm{C}}^{\mathrm{m}}(X ; r)$ for either the ambient Čech complex $\check{\mathrm{C}}^{\mathrm{m}}\left(X, \mathbb{R}^{n} ; r\right)$ or the intrinsic Čech complex $\check{\mathrm{C}}^{\mathrm{m}}(X, X ; r)$ when the distinction is not important.

Lemma 4.1. Let $\left[x_{0}, \ldots, x_{k}\right]$ be a simplex in $\check{\mathrm{C}}(X ; 2 r)$, where $X \subseteq \mathbb{R}^{n}$. Then for any $x \in \operatorname{conv}\left(\left[x_{0}, \ldots, x_{k}\right]\right)$, there exists a vertex $x_{i}$ such that $d\left(x, x_{i}\right) \leq r$.

Proof. We follow the proof of [11, Lemma 2.9] closely. By assumption, balls of radius $r$ centered at the points $x_{i}$ meet at a common point $y$. Let $x=\sum_{i} \lambda_{i} x_{i}$ be a point in $\operatorname{conv}\left(\left[x_{0}, \ldots, x_{k}\right]\right)$. Rewrite this as $\overrightarrow{0}=\lambda_{0} \hat{x}_{0}+\cdots+\lambda_{k} \hat{x}_{k}$ where $\hat{x}_{i}=x_{i}-x$. Also let $\hat{y}=y-x$. Taking the dot product with $\hat{y}$ gives

$$
0=\lambda_{0}\left\langle\hat{x}_{0}, \hat{y}\right\rangle+\cdots+\lambda_{k}\left\langle\hat{x}_{k}, \hat{y}\right\rangle .
$$

So for some $i$ we have $\left\langle\hat{x}_{i}, \hat{y}\right\rangle \leq 0$. In that case,

$$
r^{2} \geq d\left(x_{i}, y\right)^{2}=d\left(\hat{x}_{i}, \hat{y}\right)^{2}=\left\|\hat{x}_{i}\right\|^{2}-2\left\langle\hat{x}_{i}, \hat{y}\right\rangle+\|\hat{y}\|^{2} \geq\left\|\hat{x}_{i}\right\|^{2}=\left\|x_{i}-x\right\|^{2} .
$$

Lemma 4.2. For $X \subseteq \mathbb{R}^{n}$ and $r>0$, the map $f: \check{\mathrm{C}}^{\mathrm{m}}(X ; 2 r) \rightarrow \mathbb{R}^{n}$ has its image contained in $\overline{\text { Tub }}$.

Proof. For any point $x=\sum_{i} \lambda_{i} x_{i} \in \check{\mathrm{C}}^{\mathrm{m}}(X ; 2 r)$ we have that $f(x) \in \operatorname{conv}\left(\left\{x_{0}, \ldots, x_{k}\right\}\right)$. The result then follows form Lemma 4.1 .

Lemma 4.3. Let $X \subseteq \mathbb{R}^{n}$ have positive reach $\tau$, let $\left[x_{0}, \ldots x_{k}\right]$ be a simplex in $\check{\mathrm{C}}\left(X, \mathbb{R}^{n} ; 2 r\right)$ with $r<\tau$, let $x=\sum \lambda_{i} x_{i} \in \check{\mathrm{C}}^{\mathrm{m}}\left(X, \mathbb{R}^{n} ; 2 r\right)$, and let $p=\pi(f(x))$. Then the simplex $\left[x_{0}, \ldots, x_{k}, p\right]$ is in $\check{\mathrm{C}}\left(X, \mathbb{R}^{n} ; 2 r\right)$.

Proof. We write the proof for $\check{\mathrm{C}}_{\leq}\left(X, \mathbb{R}^{n} ; 2 r\right)$; an analogous proof works for $\check{\mathrm{C}}_{<}\left(X, \mathbb{R}^{n} ; 2 r\right)$. Since $\left[x_{0}, \ldots, x_{k}\right]$ is a simplex in $\check{\mathrm{C}}_{<}\left(X, \mathbb{R}^{n} ; 2 r\right)$, there exists a ball $\overline{\mathrm{B}}(y, r)$ of radius $r$ centered at some point $y \in \mathbb{R}^{n}$ such that $x_{i} \in \overline{\mathrm{B}}(y, r)$ for all $i$. Also note that $f(x) \in \operatorname{conv}\left\{x_{0}, \ldots, x_{k}\right\} \subseteq \overline{\mathrm{B}}(y, r)$. Note $p=\pi(f(x))$ is defined by Lemma 4.2. We may assume $p \neq f(x)$, since otherwise the conclusion follows from $f(x) \in \overline{\mathrm{B}}(y, r)$. Similarly, we know that $d(p, f(x)) \leq r$ since $d\left(x_{i}, f(x)\right) \leq r$ for some $i$ and since $p$ is the closest point in $X$ to $f(x)$.

Suppose for a contradiction that $p \notin \overline{\mathrm{B}}(y, r)$. Let $c=p+\tau \frac{f(x)-p}{\|f(x)-p\|}$, and let $\mathrm{B}(c, \tau)$ be the open ball with center $c$ and radius $\tau$ that is tangent to $X$ at $p$. By Proposition 2.8 every $x_{i}$ must be in $\overline{\mathrm{B}}(y, r) \backslash \mathrm{B}(c, \tau)$. Let $T_{p}^{\perp}$ be the line through $f(x)$ and $p$. We claim that $y$ cannot lie on $T_{p}^{\perp}$. Indeed if $y$ were on $T_{p}^{\perp}$ its location would be limited to one of three line segments - one with $p$ between $y$ and $f(x)$, one with $y$ between $p$ and $f(x)$, and one with $f(x)$ between $p$ and $y$. The first cannot occur as we would have $d(p, y) \leq d(f(x), y) \leq r$. The second cannot occur as we would have $d(p, y) \leq d(p, f(x)) \leq r$. Finally, the third cannot occur because either $d(p, y)<2 \tau-r$ and so the ball $\overline{\mathrm{B}}(y, r)$ is contained in $\mathrm{B}(c, \tau)$ and thus cannot contain any vertex $x_{i}$ 

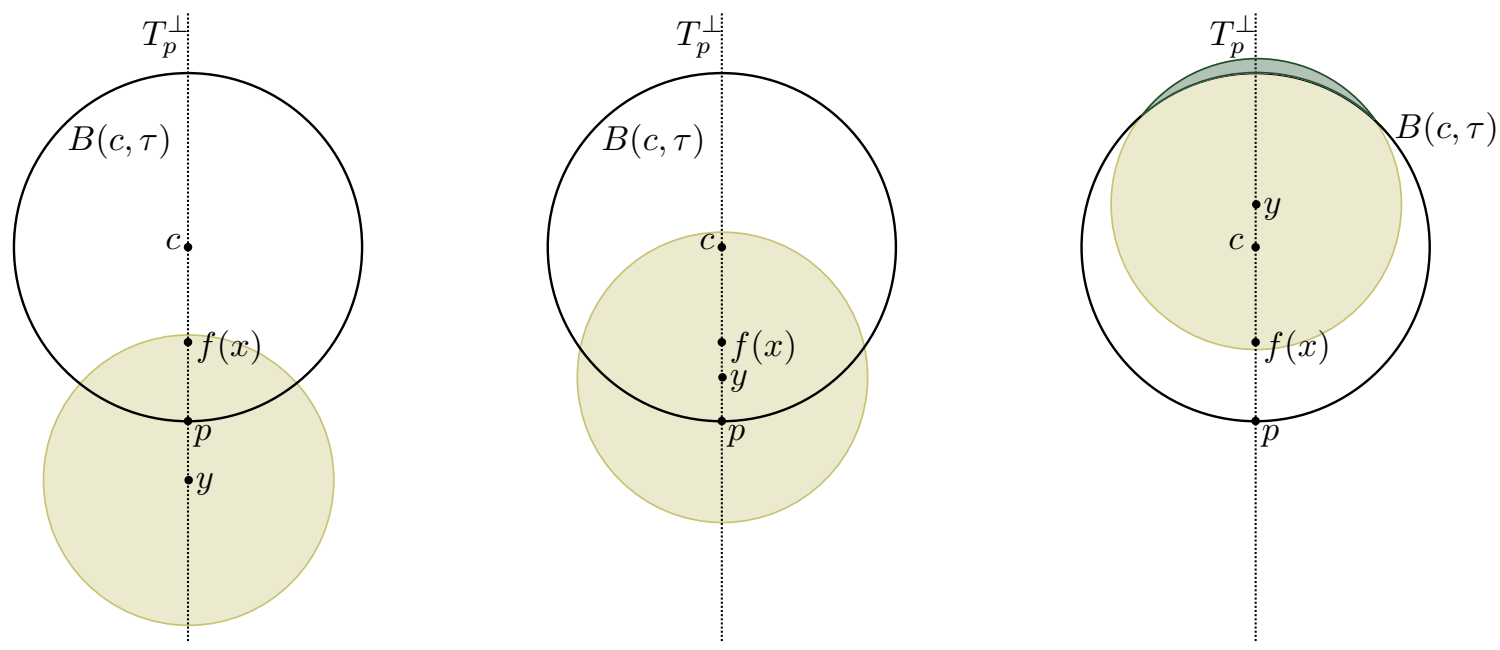

Figure 4. (Left) If $p$ is between $y$ and $f(x)$, then $\overline{\mathrm{B}}(y, r)$ contains $p$. (Middle) If $y$ is between $p$ and $f(x)$ then again $\overline{\mathrm{B}}(y, r)$ contains $p$. (Right) If $f(x)$ is between $y$ and $p$, then the green region $\overline{\mathrm{B}}(y, r) \backslash \mathrm{B}(c, \tau)$ is either empty or too far from $f(x)$.

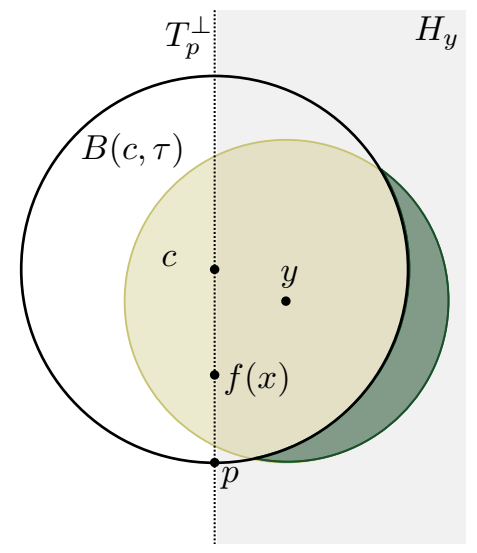

Figure 5. Figure for the proof of Lemma 4.3 . The green region $\overline{\mathrm{B}}(y, r) \backslash \overline{\mathrm{B}}(c, \tau)$ is entirely contained in $H_{y}$.

in contradiction of the definition of $y$, or $d(p, y) \geq 2 \tau-r$, in which case $d(f(x), \overline{\mathrm{B}}(y, r) \backslash \mathrm{B}(c, \tau))>r$ which contradicts Lemma 4.1 .

Let $y^{\prime} \neq y$ be the closest point on $T_{p}^{\perp}$ to $y$. Let $H_{y}=\left\{z \in \mathbb{R}^{n} \mid\left\langle z-y^{\prime}, y-y^{\prime}\right\rangle>0\right\}$ be the open half-space containing $y$, whose boundary is the hyperplane containing $T_{p}^{\perp}$ that's perpendicular to $y-y^{\prime}$. Since $f(x) \in \mathrm{B}(y, r)$ and $p \notin \mathrm{B}(y, r)$, we have $\overline{\mathrm{B}}(y, r) \backslash \mathrm{B}(c, \tau) \subseteq H_{y}$, which implies $x_{i} \in H_{y}$ for all $i$. This contradicts Lemma 3.1 since $H_{y}$ is convex with $f(x) \notin H_{y}$, even though $f(x) \in \operatorname{conv}\left(\left\{x_{0}, \ldots, x_{k}\right\}\right)$. Hence it must be the case that $p \in \overline{\mathrm{B}}(y, r)$, and so $\left[x_{0}, \ldots, x_{k}, p\right]$ is a simplex in $\check{\mathrm{C}}_{\leq}\left(X, \mathbb{R}^{n} ; 2 r\right)$.

An analogous lemma holds for the intrinsic Čech complex.

Lemma 4.4. Let $X \subseteq \mathbb{R}^{n}$ have positive reach $\tau$, let $\left[x_{0}, \ldots x_{k}\right]$ be a simplex in $\check{\mathrm{C}}(X, X ; 2 r)$ with $r<\tau$, let $x=\sum \lambda_{i} x_{i} \in \check{\mathrm{C}}^{\mathrm{m}}(X, X ; 2 r)$, and let $p=\pi(f(x))$. Then the simplex $\left[x_{0}, \ldots, x_{k}, p\right]$ is in $\check{\mathrm{C}}(X, X ; 2 r)$.

Proof. As in the ambient case, we write the proof for $\check{\mathrm{C}}_{\leq}(X, X ; 2 r)$; an analogous proof works for $\check{\mathrm{C}}_{<}(X, X ; 2 r)$. 
Since $\left[x_{0}, \ldots, x_{k}\right]$ is a simplex in $\check{\mathrm{C}}(X, X ; 2 r)$, there exists a ball $\overline{\mathrm{B}}(y, r)$ of radius $r$ centered at some point $y \in X$ such that $x_{i} \in \overline{\mathrm{B}}(y, r) \cap X$ for all $i$. Also note that $f(x) \in \operatorname{conv}\left\{x_{0}, \ldots, x_{k}\right\} \subseteq \overline{\mathrm{B}}(y, r)$, and again $p=\pi(f(x))$ is well-defined by Lemma 4.2. We may assume $p \neq f(x)$, since otherwise the conclusion follows trivially because $p \in X$ and $f(x) \in \overline{\mathrm{B}}(y, r)$, so we would have $p \in \overline{\mathrm{B}}(y, r) \cap X$. Also, we know that $d(p, f(x))<r$ since $d\left(x_{i}, f(x)\right) \leq r$ for some $i$ and since $p$ is the closest point in $X$ to $f(x)$.

Suppose for a contradiction that $p \notin \overline{\mathrm{B}}(y, r)$. Let $c=p+\tau \frac{f(x)-p}{\|f(x)-p\|}$, and let $\mathrm{B}(c, \tau)$ be the open ball with center $c$ and radius $\tau$ that is tangent to $X$ at $p$. By Proposition 2.8 every $x_{i}$ must be in $\overline{\mathrm{B}}(y, r) \backslash \mathrm{B}(c, \tau)$. As above, let $T_{p}^{\perp}$ be the line through $f(x)$ and $p$.

We now claim that $y$ cannot lie on $T_{p}^{\perp}$. Indeed, since $y \in X$, we would have either $y=p$ contradicting $p \notin \overline{\mathrm{B}}(y, r)$, or $d(y, f(x))>\tau$ because $y \notin \mathrm{B}(c, \tau)$, contradicting $f(x) \in \overline{\mathrm{B}}(y, r)$.

Let $y^{\prime} \neq y$ be the closest point on $T_{p}^{\perp}$ to $y$. Let $H_{y}=\left\{z \in \mathbb{R}^{n} \mid\left\langle z-y^{\prime}, y-y^{\prime}\right\rangle>0\right\}$ be the open half-space containing $y$, whose boundary is the hyperplane containing $T_{p}^{\perp}$ that's perpendicular to $y-y^{\prime}$. Since $f(x) \in \mathrm{B}(y, r)$ and $p \notin \mathrm{B}(y, r)$, we have $\overline{\mathrm{B}}(y, r) \backslash \mathrm{B}(c, \tau) \subseteq H_{y}$, which implies $x_{i} \in H_{y}$ for all $i$. This contradicts Lemma 3.1 since $H_{y}$ is convex with $f(x) \notin H_{y}$, even though $f(x) \in \operatorname{conv}\left(\left\{x_{0}, \ldots, x_{k}\right\}\right)$. Hence it must be the case that $p \in \overline{\mathrm{B}}(y, r) \cap X$, and so $\left[x_{0}, \ldots, x_{k}, p\right]$ is a simplex in $\check{\mathrm{C}}(X, X ; 2 r)$.

The following result is related to the nerve lemma, but it is not a consequence thereof. Indeed, even though the Čech simplicial complex $\check{\mathrm{C}}(X ; 2 r)$ is the nerve of a collection of balls, the metric Čech thickening $\check{\mathrm{C}}^{\mathrm{m}}(X ; 2 r)$ in general need not be homeomorphic nor even homotopy equivalent to the nerve $\check{\mathrm{C}}(X ; 2 r)$. We reiterate that this theorem is for both the ambient and intrinsic Cech complexes, $\check{\mathrm{C}}^{\mathrm{m}}\left(X, \mathbb{R}^{n} ; 2 r\right)$ and $\check{\mathrm{C}}^{\mathrm{m}}(X, X ; 2 r)$.

Theorem 4.5. Let $X$ be a subset of Euclidean space $\mathbb{R}^{n}$, equipped with the Euclidean metric, and suppose the reach $\tau$ of $X$ is positive. Then for all $r<\tau$, the metric Čech thickening $\breve{\mathrm{C}}^{\mathrm{m}}(X ; 2 r)$ is homotopy equivalent to $X$.

Proof. We follow the same outline as for the Vietoris-Rips case. Map $f: \check{\mathrm{C}}^{\mathrm{m}}(X ; 2 r) \rightarrow \mathbb{R}^{n}$ is again continuous by [3, Lemma 5.2]. It follows from Lemma 4.2 that the image of $f$ is a subset of Tub $_{\tau}$. By Lemma 2.7 we have that $\pi: \operatorname{Tub}_{\tau} \rightarrow X$ is continuous, and let $\iota: X \rightarrow \mathrm{VR}^{m}(X ; r)$ be the natural inclusion map.

We will show that $\iota$ and $\pi \circ f$ are homotopy inverses. Note that $\pi \circ f \circ i=\operatorname{id}_{X}$. The continuous map $H: \check{\mathrm{C}}^{\mathrm{m}}(X ; 2 r) \times I \rightarrow \check{\mathrm{C}}^{\mathrm{m}}(X ; 2 r)$ given by $H(x, t)=t \cdot \operatorname{id}_{\check{\mathrm{C}}^{\mathrm{m}}(X ; 2 r)}+(1-t) \iota \circ \pi \circ f$ is well-defined by Lemma 4.3 or 4.4 and is the necessary homotopy equivalence from $\iota \circ \pi \circ f$ to $i d_{\check{C}^{m}}(X ; 2 r)$. Hence $\check{\mathrm{C}}^{\mathrm{m}}(X ; 2 r)$ is homotopy equivalent to $X$.

In the case of the metric Čech thickening, the bound $r<2 \tau$ is tight. For example, consider the zero sphere $\mathbb{S}^{0}=\{-1,1\} \subseteq \mathbb{R}$. The reach of $\mathbb{S}^{0}$ is $\tau=1$. At scale parameter $r=2$ we have that $\check{C}_{\leq}\left(\mathbb{S}^{0}, \mathbb{R} ; 2\right) \cong[-1,1]$ is contractible, and hence not homotopy equivalent to $\mathbb{S}^{0}$.

\section{CONCLUSION}

Subsets of Euclidean space of positive reach are a class of objects of particular interest in topological data analysis, and in this paper we have shown that Vietoris-Rips and Čech thickenings of these spaces recover the same topological information as the space itself. Moreover, metric Vietoris-Rips and Čech thickenings retain metric information about the subset, in stark contrast with the classical Vietoris-Rips and Cech simplicial complexes, which in general are not metrizable. Furthermore, metric thickenings have the advantage of allowing simpler (and explicit) constructions of the maps realizing homotopy equivalences in analogues of Hausmann's result and the nerve lemma. Several questions, however, remain open. In particular, Latschev's 
theorem [21] states that if $Y$ is Gromov-Hausdorff close to a manifold $X$, then an appropriate Vietoris-Rips complex of $Y$ is homotopy equivalent to the manifold. A metric analogue for Vietoris-Rips thickenings is currently known only when $Y$ is finite ([3, Theorem 4.4]), even though we expect the result to also be true for infinite $Y$.

\section{REFERENCES}

[1] Eddie Aamari, Jisu Kim, Frédéric Chazal, Bertrand Michel, Alessandro Rinaldo, and Larry Wasserman. Estimating the reach of a manifold. arXiv preprint arXiv:1705.04565 2017.

[2] Michał Adamaszek and Henry Adams. The Vietoris-Rips complexes of a circle. Pacific Journal of Mathematics, 290:1-40, 2017.

[3] Michał Adamaszek, Henry Adams, and Florian Frick. Metric reconstruction via optimal transport. arXiv preprint arXiv:1706.04876

[4] Michał Adamaszek, Henry Adams, and Samadwara Reddy. On Vietoris-Rips complexes of ellipses. Journal of Topology and Analysis, pages 1-30, 2017.

[5] Michał Adamaszek, Florian Frick, and Adrien Vakili. On homotopy types of Euclidean Rips complexes. Discrete 63 Computational Geometry, 58(3):526-542, 2017.

[6] Dominique Attali, Jean-Daniel Boissonnat, and Herbert Edelsbrunner. Stability and computation of medial axes - a stateof-the-art report. In Mathematical foundations of scientific visualization, computer graphics, and massive data exploration, pages 109-125. Springer, 2009.

[7] Dominique Attali and André Lieutier. Geometry-driven collapses for converting a Čech complex into a triangulation of a nicely triangulable shape. Discrete \& Computational Geometry, 54(4):798-825, 2015.

[8] Gunnar Carlsson. Topology and data. Bulletin of the American Mathematical Society, 46(2):255-308, 2009.

[9] Frédéric Chazal, Vin de Silva, and Steve Oudot. Persistence stability for geometric complexes. Geometriae Dedicata, pages 1-22, 2013.

[10] Frédéric Chazal and Steve Oudot. Towards persistence-based reconstruction in Euclidean spaces. In Proceedings of the 24 th Annual Symposium on Computational Geometry, pages 232-241. ACM, 2008.

[11] Vin de Silva and Robert Ghrist. Coverage in sensor networks via persistent homology. Algebraic and Geometric Topology, 7:339-358, 2007.

[12] Tamal K Dey and Jian Sun. Normal and feature approximations from noisy point clouds. Lecture Notes in Computer Science, 4337:21-32, 2006.

[13] Herbert Edelsbrunner and John L Harer. Computational Topology: An Introduction. American Mathematical Society, Providence, 2010.

[14] David A Edwards. On the Kantorovich-Rubinstein theorem. Expositiones Mathematicae, 29(4):387-398, 2011.

[15] Herbert Federer. Curvature measures. Transactions of the American Mathematical Society, 93(3):418-491, December 1959.

[16] Charles Fefferman, Sanjoy Mitter, and Hariharan Narayanan. Testing the manifold hypothesis. Journal of the American Mathematical Society, 29(4):983-1049, 2016.

[17] Allen Hatcher. Algebraic topology. Cambridge University Press, 2002.

[18] Jean-Claude Hausmann. On the Vietoris-Rips complexes and a cohomology theory for metric spaces. In Frank Quinn, editor, Prospects In Topology, volume 138 of Annals of Mathematics Studies, pages 175-188. Princeton University Press, 1995.

[19] Hermann Karcher. Riemannian center of mass and mollifier smoothing. Communications on pure and applied mathematics, 30(5):509-541, 1977.

[20] Hans G Kellerer. Duality theorems and probability metrics. In Proceedings of the Seventh Conference on Probability theory, Braşov, Romania, pages 211-220, 1985.

[21] Janko Latschev. Vietoris-Rips complexes of metric spaces near a closed Riemannian manifold. Archiv der Mathematik, $77(6): 522-528,2001$.

[22] John M Lee. Introduction to Smooth Manifolds. Number 218 in Graduate Texts in Mathematics. Springer, 2003.

[23] Partha Niyogi, Stephen Smale, and Shmuel Weinberger. Finding the homology of submanifolds with high confidence from random samples. Discrete Computational Geometry, 39:419-441, 2008.

[24] Jan Rataj and Luděk Zajíček. On the structure of sets with positive reach. Mathematische Nachrichten, 290(11-12):18061829, 2017.

[25] Katsuro Sakai. Geometric aspects of general topology. Springer, 2013. 
[26] Edwin H Spanier. Algebraic topology, volume 55. Springer Science \& Business Media, 1994.

[27] Christoph Thäle. 50 years sets with positive reach. Surveys in Mathematics and Its Applications, 3:123-165, 2008.

[28] Anatoly Moiseevich Vershik. Long history of the Monge-Kantorovich transportation problem. The Mathematical Intelligencer, 35(4):1-9, 2013.

Department of Mathematics, Colorado State University, Fort Collins, CO 80523, United States

E-mail address: adams@math.colostate.edu

Department of Mathematics, Colorado State University, Fort Collins, CO 80523, United States

E-mail address: mirth@math.colostate.edu 